\title{
The persistence of naturally acquired antibodies and memory B cells specific to rhoptry proteins of Plasmodium vivax in patients from areas of low malaria transmission
}

Piyawan Kochayoo ${ }^{1}$, Siriruk Changrob ${ }^{1}$, Kittikorn Wangriatisak' , Seong Kyun Lee ${ }^{2}$, Patchanee Chootong ${ }^{1 *}$ and Eun-Taek Han ${ }^{2 *}$ (1)

\begin{abstract}
Background: Rhoptries are the large, paired, secretory organelles located at the apical tip of the malaria merozoite that are considered important for parasite invasion processes. Plasmodium vivax rhoptry proteins have been shown to induce humoral immunity during natural infections. Therefore, these proteins may be potential novel vaccine candidates. However, there is a lack of data on the duration of antibody and memory B cell (MBC) responses. Here, the longitudinal analysis of antibody and MBC responses to the P. vivax rhoptry proteins PVRALP1-Ecto and PvRhopH2 were monitored and analysed in individuals to determine their persistence.

Methods: Thirty-nine samples from P. vivax-infected subjects (age 18-60 years) were recruited to explore the frequency and persistence of antibody and MBC responses against rhoptry proteins (PVRALP1-Ecto and PvRhopH2) using both cross-sectional and longitudinal cohort study designs. Antibody levels were determined by ELISA during clinical malaria, and at 3,9 and 12 months post-infection. The frequency of MBC sub-sets and presence of rhoptryspecific MBCs in subjects 18 months after treatment were detected by flow cytometry and ELISPOT assay.

Results: The seroprevalence of antibodies against PVRALP1-Ecto and PVRhopH2 proteins was found to be high during acute infection, with $\lg G 1$, lgG2 and lgG3 sub-classes predominant. However, these anti-rhoptry responses were short-lived and significantly decreased at 9 months post-infection. To relate the durability of these antibody responses to MBC persistence at post-infection, 18-month post-infection peripheral blood mononuclear cells (PBMCs) samples were taken to detect rhoptry-specific MBCs and frequency of MBC sub-sets, and correlate with antibody responses. These late post-infection samples revealed that rhoptry-specific MBCs were present in about $70 \%$ of total subjects. However, the persistence of specific MBCs was not correlated with antibody responses as the majority of malaria subjects who were positive for PvRALP1-Ecto- or PvRhopH2-specific MBCs were seronegative for the rhoptry antigens. The frequencies of classical MBCs were increased after infection, whereas those of activated and atypical MBCs were decreased, indicating that MBC responses could switch from activated or atypical MBCs to classical MBCs after parasite clearance, and were maintained in blood circulating at post-infection.
\end{abstract}

\footnotetext{
*Correspondence: pchooton@gmail.com; ethan@kangwon.ac.kr

${ }^{1}$ Department of Clinical Microbiology and Applied Technology, Faculty

of Medical Technology, Mahidol University, Bangkok 10700, Thailand

${ }^{2}$ Department of Medical Environmental Biology and Tropical Medicine,

School of Medicine, Kangwon National University, Chuncheon,

Gangwon-do 200-701, Republic of Korea
}

C The Author(s) 2019. This article is distributed under the terms of the Creative Commons Attribution 4.0 International License (http://creativecommons.org/licenses/by/4.0/), which permits unrestricted use, distribution, and reproduction in any medium, provided you give appropriate credit to the original author(s) and the source, provide a link to the Creative Commons license, and indicate if changes were made. The Creative Commons Public Domain Dedication waiver (http://creativecommons.org/ publicdomain/zero/1.0/) applies to the data made available in this article, unless otherwise stated. 
Conclusion: The study showed that rhoptry antigens induced the development and persistence of MBC responses in P. vivax-infected subjects who lived in a region of low malaria transmission, which were not related to the longevity of antibody responses.

Keywords: Plasmodium vivax, PvRALP1-Ecto, PvRhopH2, Antibody responses, Memory B cells

\section{Background}

Malaria remains an important global health issue causing illness and death worldwide, especially in tropical and sub-tropical areas. There were an estimated 219 million malaria cases and more than 400,000 deaths in 2017 [1]. Plasmodium vivax is the most prevalent cause of recurring malaria and has a wide geographic distribution, mainly in Asia-Pacific and Latin American regions [2]. Relapse is a unique feature of $P$. vivax biology, making it difficult to control because the parasite can be hidden for a long time in the patient's liver and then emerge to cause relapsing $P$. vivax $[3,4]$. Given the difficulties with treatment, diagnosis and control, an effective vaccine would be valuable in eliminating and preventing the disease [4].

There is currently no licensed vaccine available for malaria. Several antigen candidates against Plasmodium falciparum have moved into clinical trials. In contrast, $P$. vivax vaccine development has lagged far behind, as only three $P$. vivax vaccine candidates (PvDBP, PvCSP, Pvs25) have even reached phase I clinical trials [5-7]. This may reflect previous neglect of $P$. vivax, technical challenges such as maintaining $P$. vivax in culture, and limited animal models of infection. For blood-stage malaria vaccines, the aim is to block parasite invasion and subsequently reduce the clinical burden. At present, $P$. vivax Duffy-binding protein region II (PvDBPII) is a leading vaccine candidate because $P$. vivax invasion of erythrocytes is largely dependent upon its interaction with the Duffy blood-group antigen [8]. It induces antibody responses in populations naturally exposed to $P$. vivax and protects against high-density $P$. vivax infection by inhibiting parasite invasion into red blood cells (RBCs) [9]. However, high polymorphism of this micronemal protein is a major challenge in designing a vaccine that will produce protective immunity responses against the conserved epitopes against a panel of variant P. vivax isolates [10]. Moreover, vivax malaria in Duffynegative individuals in Africa was recently reported, indicating that there are Duffy antigen/chemokine receptor (DARC)-independent pathways for $P$. vivax invasion [11]. Thus, a novel parasite ligand is required for parasite invasion. Such a protein, which has immunogenicity to elicit immune responses that will block merozoite invasion of RBCs and stop rapid replication of merozoites, has been identified.
Antibody responses to blood-stage malaria are required for inhibition of parasite invasion [12-15]. Longitudinal studies of humans living in areas of high malaria transmission showed that repeated $P$. falciparum infections can induce antibody responses to blood-stage antigens but that these responses were relatively short-lived [16]. Likewise, the antibody profiles in malaria-naïve and semi-immune Colombian volunteers experimentally infected with $P$. vivax were short-lived and had returned to near baseline by day 145 [17]. The presence of these antibody responses was independent of the presence of malaria-specific memory B cells (MBCs), since individuals residing in areas of low transmission have been shown to generate stable Plasmodium-specific MBCs without frequent boosting $[18,19]$. However, children and young adults in an area of high seasonal transmission in Mali demonstrated delayed development of $P$. falciparum-specific $\mathrm{MBCs}$ despite repeated infections annually $[20,21]$. This evidence indicates that infection rates and the diverse genetics of both parasite and host could be involved in the development and maintenance of malariaspecific MBCs [22-24]. Therefore, better understanding of the durability of both antibody and $\mathrm{MBC}$ responses in patients with naturally acquired malaria, as well as identifying the antigens that induce these long-term responses, will be useful in the development of protective bloodstage malaria vaccines.

The rhoptry is a large secretory organelle of the merozoite that contains a wide range of proteins which are involved in invasion of RBCs [25, 26]. It is composed of at least two sub-domains, referred to as the rhoptry neck and the rhoptry bulb. Several rhoptry proteins, such as $P$. falciparum rhoptry neck protein (RON) 2-4, are involved in tight junction formation between the parasite and its target cells by interacting with the micronemal protein apical membrane antigen (AMA) 1 [27, 28]. However, some rhoptry proteins are released during invasion and migrate to the lumen or membrane of the parasitophorous vacuole [29]. The rhoptry-associated leucine zipper-like protein 1 (RALP1) and high-molecular-weight complex rhoptry proteins (RhopH) have been characterized as being crucial during $P$. falciparum infection [30,31]. These two proteins are localized in the rhoptry of $P$. falciparum merozoites [30, 32]. RALP1 possesses a leucine zipper-like domain that facilitates protein-protein interaction [30] and $\mathrm{RhopH} 2$ contains 
a signal peptide at its $\mathrm{N}$-terminal and 12 cysteines in its C-terminal half [33]. Furthermore, these rhoptry proteins are conserved in Plasmodium spp. [34], suggesting that they may be involved in parasite invasion.

High antigenicity of rhoptry proteins has often been reported in malaria patients [33, 35-37]. The rhoptry neck protein of merozoites, PvRALP1, triggers IgG3, IgG2 and IgG1 isotype responses in P. vivax-exposed Korean individuals, and purified serum anti-PfRALP1 from Malian $P$. falciparum-infected patients inhibits parasite invasion [30, 35]. Two $P$. vivax proteins at the rhoptry bulb, rhoptry-associated membrane protein (RAMA) and RhopH2 antigens, show an ability to induce the acquisition of humoral immunity in both mouse models and patients [33, 38]. Interestingly, the anti-PvRAMA response is maintained up to 9 months after anti-malarial treatment, and some patients maintain antibody responses up to 12 months post-infection [38]. All of these data indicate that the combination of these rhoptry proteins with other blood-stage proteins in a vaccine design may induce protective responses of humoral immunity. However, due to poor understanding of the immune responses to rhoptry proteins and the duration of these responses, more data are required from immuno-epidemiological studies on naturally acquired MBCs and antibody responses. Thus, in the present study two rhoptry protein antigens, PvRALP1-Ecto and PvRhopH2, were used to characterize the long-term responses of antibodies and MBCs in P. vivax patients living in a low-malaria-endemicity region of southern Thailand. Longitudinal sampling of participants in a cohort study was conducted to determine whether individuals exposed to $P$. vivax developed and were able to maintain antibody and $\mathrm{MBC}$ responses to these rhoptry proteins.

\section{Methods}

\section{Study site and sample collection}

This study was conducted in low-malaria-transmission areas of southern Thailand (Chumphon Province) where both $P$. falciparum and $P$. vivax were common. Both a cross-sectional survey and a cohort study were set up between 2014 and 2017 to explore the antigenicity of rhoptry proteins and monitor the longevity of antibody and $\mathrm{MBC}$ responses. First, serological responses to rhoptry proteins were determined from 39 patients with acute P. vivax infection (Table 1). To observe the development and maintenance of antibody and $\mathrm{MBC}$ responses, only individuals with samples available from all follow-up time points were used (18 subjects; Table 2). Subsequently, the samples were analysed for total IgG and subtype IgG as detected by ELISA (sub-cohort 1 study).

To explore the basis of persistence of humoral immunity against rhoptry antigens in P. vivax infection, the
Table 1 Characteristics of study participants in survey of PvRhoptry antigenicity during acute Plasmodium vivax infection

\begin{tabular}{lll}
\hline Characteristic & Acute vivax patients & Healthy subjects \\
\hline Total (n) & 39 & 27 \\
Gender & & \\
Male & 27 & 8 \\
Female & 12 & 19 \\
Ages (years) & $40.1 \pm 11.4$ & $20.2 \pm 0.8$ \\
Mean \pm SD (range) & $(18-60)$ & $(19-21)$ \\
Seropositive responders & & \\
PvRALP1-Ecto & 26 & 0 \\
PvRhopH2 & 29 & 0 \\
Both PvRALP1 and & 22 & 0 \\
$\quad$ PvRhopH2 & & \\
Parasitaemia (parasite/ $\mu \mathrm{L})$ & $5170.8 \pm 5238.3$ & 0 \\
Mean \pm SD (range) & $(200-15,000)$ & \\
\hline
\end{tabular}

kinetics of B cell sub-sets in the acute and recovery phases, and the persistence of rhoptry-specific MBCs were investigated in the sub-cohort 2 study (May 2016 to May 2018). Peripheral blood mononuclear cells (PBMCs) from 10 individuals were isolated to perform for flowcytometric analysis and ELISPOT assays. Plasma was collected for detection of total IgG and IgG subclasses. Peripheral blood samples $(10 \mathrm{~mL})$ from participants were obtained by venipuncture into heparinized tubes before administration of treatment, and transported to the laboratory for processing within $6 \mathrm{~h}$ of collection. The PBMCs were separated by Ficoll-Hypaque density-gradient centrifugation and used for MBC experiments. Plasma was stored at $-20^{\circ} \mathrm{C}$ for antibody analyses.

The inclusion criteria for subject recruitment were the following: (1) systolic blood pressure not less than $90 \mathrm{mmHg}$; (2) body temperature not higher than $40{ }^{\circ} \mathrm{C}$; (3) haematocrit not less than $25 \%$; (4) no prior treatment with corticoids or non-steroidal anti-inflammatory drugs (NSAIDs); and, (5) age of 18 years or above. Those who did not meet the criteria were excluded. Written informed consent was obtained from all participants before enrollment. The $P$. vivax infection was diagnosed by microscopic examination of both thin and thick blood films, and confirmed by nested PCR. Data on occurrence and number of prior $P$. vivax infections in each individual were obtained from the records of the local Vector-borne Disease Unit 11.4.2. The malaria staff collected posttreatment blood samples from patients every 3 months to check for sub-clinical malaria by nested PCR. To estimate the incidence of clinical malaria over the study period, malaria staff had carried out weekly house-to-house visits from May 2014 to May 2017, and in May 2018, with confirmation of infection by nested PCR. Malaria-unexposed 
Table 2 Characteristics of participants $(n=18)$ in the study of total IgG and IgG isotype longevity

\begin{tabular}{|c|c|c|c|c|c|c|c|}
\hline \multirow[t]{2}{*}{ By antigen } & \multirow{2}{*}{$\begin{array}{l}\text { No. positive responders } \\
\text { at acute phase }\end{array}$} & \multirow{2}{*}{$\begin{array}{l}\text { Age } \\
\text { Mean } \pm S D\end{array}$} & \multicolumn{2}{|c|}{ Gender } & \multicolumn{2}{|c|}{ Nationality } & \multirow{2}{*}{$\begin{array}{l}\text { No. recorded } \\
\text { re-infections }\end{array}$} \\
\hline & & & Male & Female & Thai & Myanmar & \\
\hline PV-RALP1-Ecto & 14 & $44.2 \pm 9.7$ & 13 & 5 & 17 & 1 & 1 \\
\hline PvRhopH2 & 14 & $44.2 \pm 9.7$ & 13 & 5 & 17 & 1 & 1 \\
\hline
\end{tabular}

persons living in Bangkok, Thailand, were recruited as healthy controls $(\mathrm{HC}, \mathrm{n}=27)$. Ethical approvals were obtained from the Committee on Human Rights Related to Human Experimentation, Mahidol University, Thailand (MUIR 2012/079.2408).

\section{Expression and purification of recombinant PvRALP1-Ecto and PvRhopH2 proteins}

To express the recombinant PvRALP1-Ecto and PvRhopH2 proteins, the protocol followed that of previous studies [33, 35]. In brief, the open reading frame of pvralp1-ecto, comprising amino acids 31 to 675 without the signal peptide sequence, and the first exon of the pvrhoph 2 sequence, amino acid position 67 to 1161, were amplified with the following primers: PvRALP1-Ecto F: $5^{\prime}$-atcactagttctcgagATGGCGTACCGCCTAAAGAGG$3^{\prime}$ and the reverse primer PvRALP1-Ecto R: $5^{\prime}$-ccctatatatggatccTCACTAGAACATGTCGTAGAGCAGGC-3', RhopH2 F:PvRhopH2 F: 5'-GGGCGGATATCTCGA GGAGCTGAGCCACAGCTTGT- ${ }^{\prime}$ and PvRhopH2 R: 5'-GCGGTACCCGGGATCCTCACT TCTCCACAT CCTCGTGGT-3'. The purified PCR products were then cloned into the $\mathrm{XhoI}$ and $\mathrm{BamHI}$ sites of the pEUE01-His-Tev-N2 plasmid vector, which is an expression plasmid with an N-terminal hexa-histidine (His)-tag followed by a tobacco etch virus protease cleavage site. Plasmid DNA was prepared, purified and eluted in $0.1 \times \mathrm{TE}$ buffer (10 mM Tris-HCl, pH 8.0, 1 mM EDTA), and used for in vitro transcription. The recombinant proteins were synthesized with a wheat germ cell-free protein expression system (CellFree Sciences, Matsuyama, Japan), using the bilayer translation reaction method as described previously [39]. The His-tagged recombinant proteins were purified using a Ni-Sepharose column (GE Healthcare, Camarillo, CA, USA) and eluted with phosphate-buffered saline (PBS) containing $500 \mathrm{nM}$ imidazole. Finally, the purified recombinant proteins PvRALP1-Ecto and PvRhopH2 were detected by SDS-PAGE and Western blot analyses.

\section{Measurement of total IgG and IgG subtype responses}

Naturally acquired antibodies against recombinant PvRALP1-Ecto and PvRhopH2 were detected by ELISA. In brief, 96-well, flat-bottom microplates (Corning, Corning, NY, USA) were pre-coated with $0.5 \mu \mathrm{g}$ recombinant
PvRALP1-Ecto or PvRhopH2 per well and incubated overnight at $4{ }^{\circ} \mathrm{C}$. After blocking with PBS containing $4 \%$ BSA for $2 \mathrm{~h}, 100 \mu \mathrm{L}$ of each plasma sample at a dilution of 1:200 was added to duplicate wells and incubated at room temperature for $1 \mathrm{~h}$. The plates were washed with PBS containing $0.05 \%$ Tween 20 (PBS-T) five times and incubated with peroxidase-conjugated, goat anti-human IgG (Sigma, St. Louis, MO, USA) for $1 \mathrm{~h}$. Subsequently, washing was repeated, and the plates were developed with 2,2'-azino-bis (3-ethylbenzthiazoline-6-sulphonic acid) substrate (R\&D Systems, Minneapolis, MN, USA) for $45 \mathrm{~min}$. The optical density (OD) was determined at $405 \mathrm{~nm}$ using a plate reader. The positive samples $(\mathrm{n}=2)$ were $P$. vivax plasma that showed seropositive to $\mathrm{rDB}$ PII antigen from previous study [40]. The OD values for duplicate wells per individual were averaged. A baseline OD was established using plasma samples from 27 healthy individuals living in non-malaria endemic areas $(\mathrm{HC})$.

IgG isotype responses to rhoptry proteins were detected following a protocol similar to that for total IgG detection. In the step when each plasma sample was added at a dilution of 1:100, followed by addition of conjugated antibodies, horseradish peroxidase (HRP)-conjugated anti-human IgG1, IgG2, IgG3, and IgG4 antibodies at a dilution of 1:1000 were used for detection. The OD of the reaction was measured at $450 \mathrm{~nm}$ after the addition of the tetramethylbenzidine (TMB) enzyme-substrate. The positive controls were plasma $(n=2)$ of the vivax malaria patient who had the highest OD value for total IgG. A baseline OD was established using plasma samples from 20 healthy individuals living in non-malaria endemic areas $(\mathrm{HC})$.

The levels of total IgG or IgG subclasses specific to PvRALP1-Ecto or PvRhopH2 were expressed as reactivity indexes (RI), which were obtained by dividing the mean sample OD by the cut-off value. The cut-off value (mean OD +2 standard deviations (SDs)) was calculated from a set of negative controls obtained from plasma samples from HC. A sample was considered to be positive for a specific antibody if the RI was $\geq 1$.

\section{Memory B cell phenotyping}

PBMCs collected during acute malaria and 18 months post-infection were used for B cell sub-set phenotyping. Fluorochrome-conjugated, mouse anti-human 
monoclonal antibodies were used to stain 1 million PBMCs $/ 100 \mu \mathrm{L}$ FACS buffer. A cocktail consisting of the following mouse monoclonal antibodies was used: FITCCD19, PerCP-CD20, PE/Cy7-CD10, PE-CD27, and APCCD21 (Biolegend, San Diego, CA, USA). After staining for $15 \mathrm{~min}$, cells were washed with FACS buffer. Finally, cells were suspended in $250 \mu \mathrm{L}$ FACS buffer. The analyses were done by a flow cytometer (BD FACSCanto II, Becton-Dickinson Immunocytometry Systems, San Jose, CA, USA). Data were processed using FlowJo software (Tree Star, San Carlos, CA, USA).

\section{B cell ELISPOT assay}

The presence of rhoptry-specific MBCs was determined by ELISPOT assay as performed in a previous study [41]. In brief, $9 \times 10^{6}$ PBMCs from $P$. vivax subjects were prepared for cell stimulation along with negative controls. First, $1 \times 10^{6}$ cells per $\mathrm{mL}$ were stimulated with polyclonal activator R848 and recombinant human IL-2 (Mabtech $\mathrm{AB}$, Stockholm, Sweden) at $37^{\circ} \mathrm{C}$ in a humidified $5 \% \mathrm{CO}_{2}$ incubator for $72 \mathrm{~h}$. Then, the ELISPOT assay was performed by coating with $15 \mu \mathrm{g} / \mathrm{mL}$ of monoclonal antibodies of anti-human IgG (clones MT91/145; Mabtech), $5 \mu \mathrm{g} / \mathrm{mL}$ of recombinant PvRALP1-Ecto or PvRhopH2 rhoptry antigens, or $1 \mu \mathrm{g} / \mathrm{mL}$ of tetanus toxoid (TT) antigen (Merck Millipore, Darmstadt, Germany) onto MultiScreen Filter PVDF Immobilon plates (Merck Millipore). Cells were harvested and seeded in duplicate to yield $5 \times 10^{4}$ cells per anti-human IgG-coated well, and $1 \times 10^{6}$ cells per specific antigen-coated well. After $24 \mathrm{~h}$ of incubation at $37{ }^{\circ} \mathrm{C}$ under $5 \% \mathrm{CO}_{2}, 1 \mu \mathrm{g} / \mathrm{mL}$ of detection monoclonal antibodies MT78/145 (Mabtech) diluted in PBS-0.5\% FBS was added and incubated for $2 \mathrm{~h}$ at room temperature. Following a thorough washing with PBS, the diluted (1:1000) streptavidin-HRP-conjugated, polyclonal goat anti-human IgG (Mabtech) and TMB substrate for ELISPOT (Mabtech) were added. The filtered plates were rinsed with deionized water after distinct spots emerged. Antigen-specific MBCs were expressed as spot-forming cells (SFCs) in the wells. The plates were analysed with a Bioreader 5000 Pro-F gamma ELISPOT Reader (BioSys GmbH, Karben, Germany). PBMCs from P. vivaxinfected subjects that were cultured without stimulation and incubated overnight with rhoptry antigens were used as negative controls. A positive ELISPOT response was defined as detectable spots in duplicate wells with the total spots in the specific antigen-coated wells being at least twice the number of spots detected with the negative control samples.

\section{Statistical analyses}

The Mann-Whitney U test was used to compare data of 2 groups of samples (unpaired) from different subjects, while the Wilcoxon signed rank test was used to compare data of 2 groups of paired data from the same subjects at different time points. Kruskal-Wallis one-way ANOVA was used to compare data from more than 2 groups. The association between the responses of rhoptry-specific MBCs by ELISPOT and antibodies by ELISA was done using Fisher's exact tests. In all analyses, 2-tailed $P$-values $<0.05$ were considered significant. The statistical analysis was performed and graphs prepared using GraphPad Prism software (Systat Software, San Jose, CA, USA).

\section{Results}

\section{Sample organization}

There are three distinct groups of data in this study (Fig. 1). First, the cross-sectional survey was conducted in $39 P$. vivax-infected patients, using plasma samples collected from May 2014 to May 2017 (Table 1). Some of these individuals dropped out of some sample collections. From the total 39 infected subjects, blood samples were available at all time points from 18 subjects. These 18 subjects were studied as 'sub-cohort 1' to explore the longevity of antibody responses (both total IgG and IgG isotype levels) against PvRALP1-Ecto and PvRhopH2 antigens (Table 2). Furthermore, to assess the relationship between persistence of detectable antibody and MBC responses post-infection, 'sub-cohort 2' was created by recruiting $10 P$. vivax-infected patients between May 2016 and May 2018 to determine MBC sub-set responses and the persistence of PvRALP1-Ecto- and PvRhopH2specific MBCs at 18 months post-infection.

\section{Antigenicity of rhoptry antigens in acute and recovery phases of Plasmodium vivax infection}

To survey the antigenicity of rhoptry proteins in natural exposure to $P$. vivax parasites, the antibody titres against recombinant PvRALP1-Ecto and PvRhopH2 antigens were determined during infection using a cross-sectional survey. In the acute phase, anti-rhoptry responses were significantly higher than those in healthy controls (PvRALP1-Ecto; P $<0.0001$, PvRhopH2; $\mathrm{P}<0.0001$ ). Subject positivity to PvRALP1-Ecto and PvRhopH2 was 66.67\% (26 of 39) and 29 74.36\% (29 of 39), respectively (Fig. 2). Among PvRALP1-Ecto and PvRHopH2 seropositive responders, there were 22 patients who were antibody positive against both rhoptry proteins (Table 1). Thus, the longevity of antibody responses against rhoptry proteins was further explored in the sub-cohort 1 study.

\section{Persistence of anti-rhoptry IgG specific responses after acute infection with Plasmodium vivax}

To determine the durability of anti-rhoptry responses after infection, antibody titres were measured in the 18 


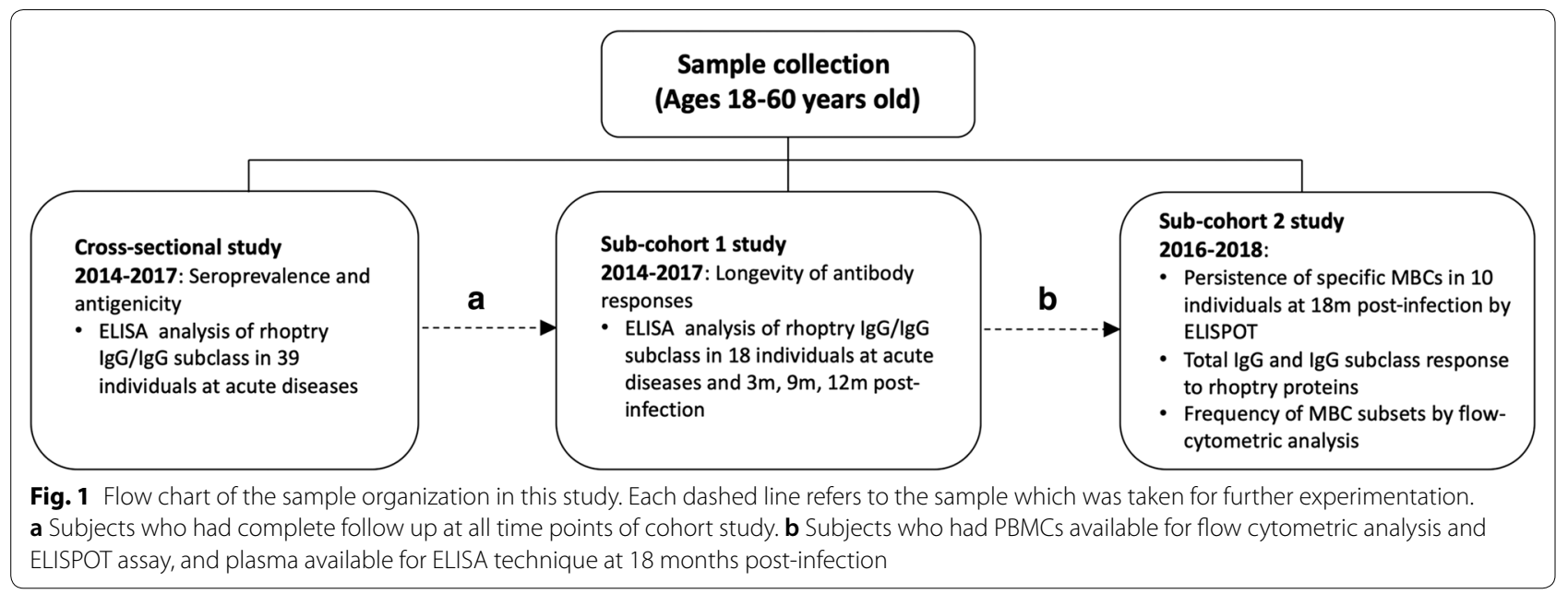

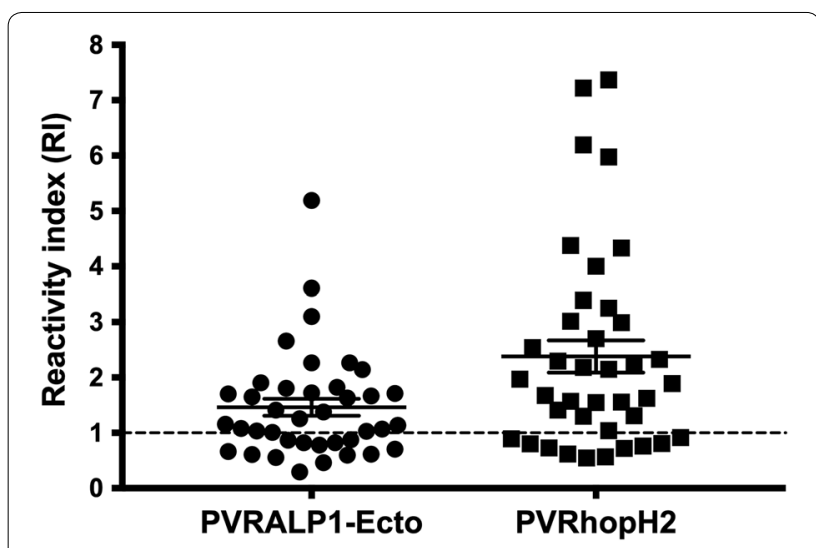

Fig. 2 The seroprevalence of anti-IgG rhoptry responses. The reactive index (RI) of specific lgG responses to PVRALP1-Ecto and PVRhopH2 antigens in the plasma of acute $P$. vivax-infected patients $(n=39)$ was detected ELISA. The horizontal line represents mean values \pm SEM. Dashed line indicates the cut-off value $(R I \geq 1)$

individuals who were monitored at all time points of the cohort study (acute, 3, 9 and 12 months). Both antiPvRALP1-Ecto and anti-PvRhopH2 titres were significantly reduced at 9 months post-infection (Fig. 3a). Only 38.89 and $77.78 \%$ of patients showed a positive response to PvRALP1-Ecto and PvRhopH2 antigens, respectively, at 9 months post-infection (Fig. 3a). Interestingly, the monitoring kinetic of PvRALP1-Ecto and PvRhopH2 responses up to 12 months' post-infection showed that 5 and 10 patients, respectively, stably maintained their antibody responses (Fig. 3b, c). Thus, the IgG isotypes which played major roles in the humoral immunity against rhoptry protein was further explored in acute vivax malaria and after infection.

\section{IgG2 isotype had a stable response post-infection}

As different IgG sub-classes have the ability to provide protective immunity $[42,43]$, the predominant anti-IgG rhoptry antibody isotype was determined in the acute and recovery phases of these $P$. vivax infections. In the acute phase, the prevalent rates of anti-PvRALP1-Ecto IgG sub-classes were $79.49 \%$ for IgG1, 76.92\% for IgG2, $69.23 \%$ for IgG3, and $53.85 \%$ for IgG4. In case of antiPvRhopH2 IgG subclass responses, the IgG1, IgG2, IgG3, and IgG4 frequency distribution of individuals that recognized the antigen was $64.10 \%, 58.97 \%, 66.67 \%$, and $43.59 \%$, respectively (Fig. 4a). Therefore, the predominant sub-class response to PvRALP1-Ecto was IgG1 and IgG2 whereas a higher seroprevalence of IgG1 and IgG3 responders was shown in PvRhopH2. A further analysis of IgG isotype responses during acute malaria and 9 months post-infection in sub-cohort 2 subjects showed that IgG1 and IgG2 response to PvRALP1-Ecto at 9 months post-infection was not significantly different compared to those during the acute phase (Fig. 4b). However, a greater decrease of IgG3 responses to these two rhoptry protein was shown at 9 months post-infection (Fig. 4b, c).

\section{Memory B cells specific to rhoptry proteins were detected at least 18 months post-infection}

As some patients showed the persistence of anti-rhoptry antibodies for at least 12 months, this could be due to the ability of rhoptry antigens to induce $\mathrm{MBC}$ development during acute malaria and to maintain it after recovery from infection. The sub-cohort 2 study was designed to explore the presence of rhoptry-specific $\mathrm{MBC}$ responses during the recovery phase (see Additional file 1). Of the 10 subjects, 7 and 9 had MBCs to PvRALP1-Ecto 

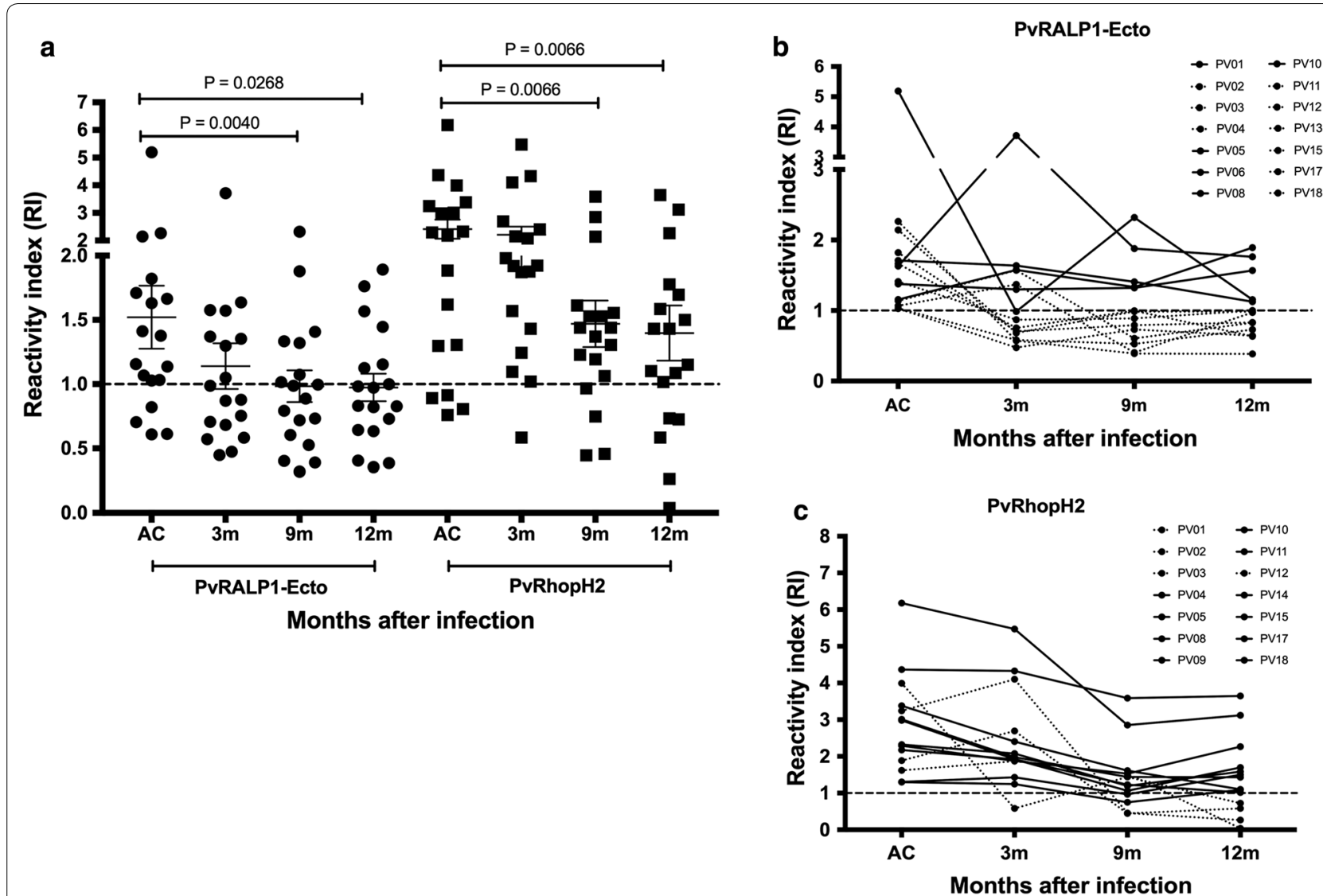

Fig. 3 Longitudinal analysis of anti-rhoptry responses. The reactive index (RI) of specific IgG responses to PVRALP1-Ecto and PVRhopH2 antigens in the plasma of P. vivax subjects during follow-up was detected by ELISA. a Anti-PVRALP1-Ecto and anti-PVRhopH2 responses at all time points in the cohort study: acute infection and 3, 9, and 12 months after infection $(n=18)$. The horizontal line represents mean values \pm SEM. $\mathbf{b}$, $\mathbf{c}$ The kinetics of antibody responses against PVRALP1-Ecto and PVRhopH2 in 14 seropositive P. vivax-infected patients at different time points: acute infection and 3,9 , and 12 months after infection. Solid lines indicate individual subjects who have antibody responses to PvRALP1-Ecto and PvRhopH2 up to 12 months. Dotted lines indicate individual subjects who have antibody responses to PvRALP1-Ecto and PvRhopH2 less than 12 months. Dashed line indicates the cut-off value (RI $\geq 1)$

and PvRhopH2 antigens, respectively (Fig. 5a). The PvRALP1-Ecto- and PvRHopH2-specific MBCs showed spot forming cells (SFCs) in the ranges of 2-19 and 2-29, respectively. As expected, all patients produced TTspecific MBCs (range, 4-49 SFCs). A statistical analysis of association between the responses of rhoptry-specific MBCs and antibodies in individuals at 18 months postinfection showed that of 7 individuals positive for MBCs to PvRALP1-Ecto, 6 were seronegative $(P$ value $=1.00)$; of 9 individuals positive for MBCs to PvRhopH2, 6 were seronegative $(P$ value $=0.40)$ (Fig. $5 \mathrm{~b}$ ). In addition, the seronegative levels of anti-PvRALP1-Ecto and -PvRhopH2 IgG were related with the short-lived of IgG sub-class response at 18 months post-infection patients. Of total 10 samples, only 3, 1 and 2 patients maintained seropositive for anti-PvRALP1-Ecto-IgG2, antiPvRhopH2-IgG1 and anti-PvRhopH2-IgG2 responses, respectively (Fig. 5c).

\section{$B$ cell surface marker analysis showed functional of MBC} responses

To characterize the $\mathrm{MBC}$ sub-sets in relation to the responses of rhoptry-specific MBCs after $P$. vivax infection, activated, atypical and classical MBCs were determined by phenotyping PBMC samples from both acute phase and at 18 months post-infection. Of the 10 patients in the sub-cohort 2 study, 9 had available PBMCs for MBC sub-set phenotyping by flow cytometric analysis. In acute infection, the frequencies of the activated and atypical MBCs were significantly elevated, whereas classical MBCs were significantly reduced compared to those of healthy controls (Fig. 6a-c). In contrast, activated and atypical MBCs were decreased and classical MBCs increased at 18 months post-infection (activated MBC: average $2.43 \%$, SD $1.32 \%$; atypical MBCs: average $4.37 \%$, SD 1.67\%; classical MBCs: average 56.88\%, SD 15.34\%) (Fig. $6 \mathrm{a}-\mathrm{c}$ ). These data indicated that classical MBCs 

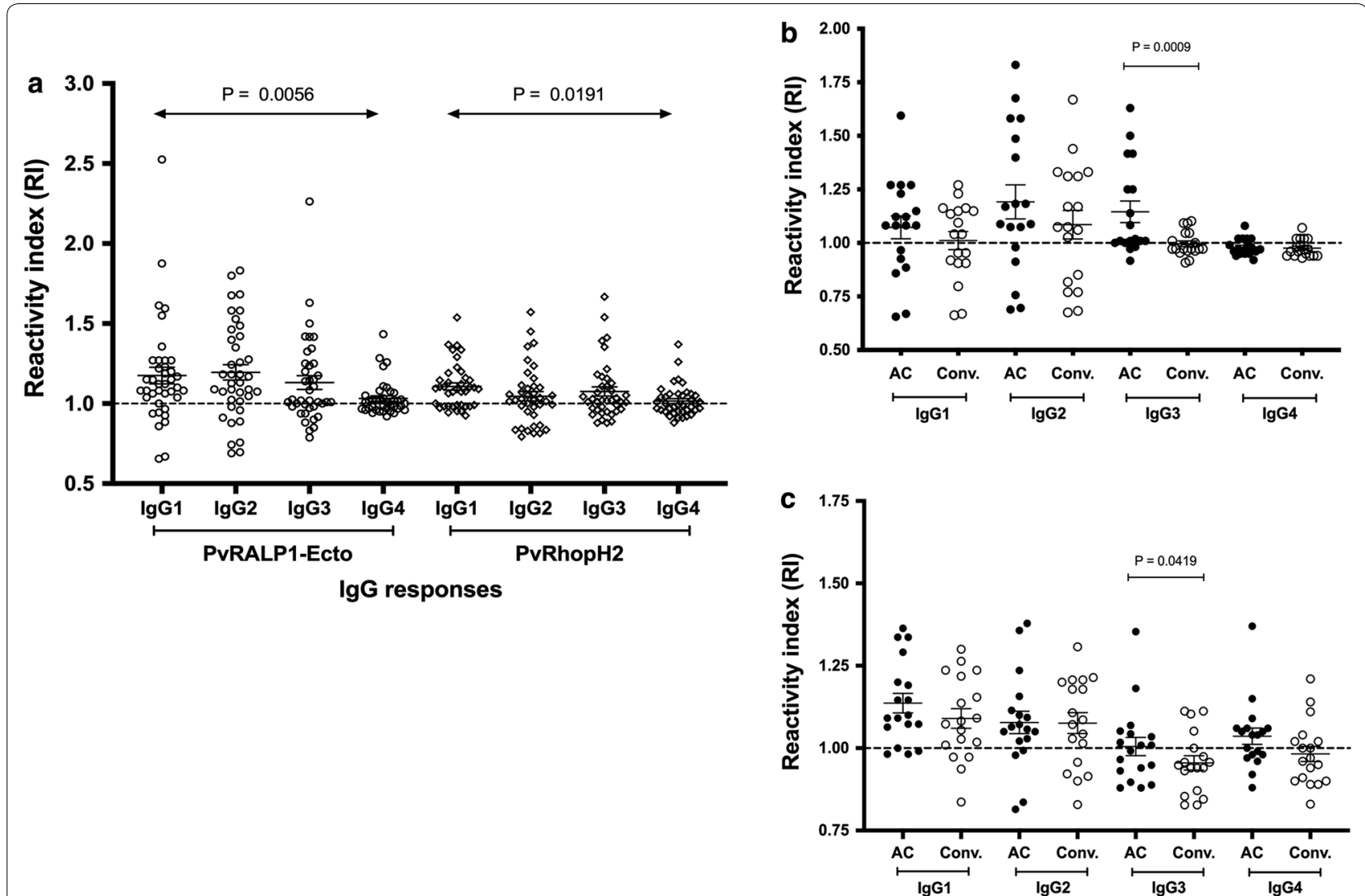

Fig. 4 The prevalence of IgG sub-class responses to rhoptry proteins. The reactive index (RI) of specific lgG sub-class responses to PvRALP1-Ecto and PVRhopH2 antigens in the plasma of P. vivax subjects at acute and during follow-up was detected by ELISA. a The comparative analysis of anti-lgG1, -lgG2, -lgG3, and -lgG4 specific to PvRALP1-Ecto and PvRhopH2 proteins in individuals with acute P. vivax malaria $(A C, n=39)$. The Kruskal-Wallis one-way ANOVA test was performed to compare the mean antibody levels of the difference lgG sub-classes. The horizontal line represents mean values \pm SEM. $\mathbf{b}, \mathbf{c}$ The anti-lgG1, $\lg G 2, \lg G 3$ and $-\lg G 4$ responses to these two rhoptry proteins in 18 P. vivax subjects in the acute phase (AC) and convalescence phase (Conv; 9 months post-infection). The comparisons of IgG sub-class response in the plasma of subjects during follow up ( $A C$ and Conv., $\mathrm{n}=18$ ) were done by Wilcoxon signed rank tests. Dashed line indicates the cut-off value $(R I \geq 1)$

were the only $\mathrm{MBC}$ sub-set which persisted at 18 months post-infection and maintained positive MBCs specific for rhoptry proteins.

\section{Discussion}

It has long been known that individuals living in areas of high malaria endemicity are susceptible to re-infection because their anti-malarial antibodies are shortlived [20, 21]. However, antibody responses to malaria infection develop more effectively in low transmission areas, even after only one or two documented $P$. falciparum infections $[18,19]$. Here, two rhoptry proteins of the P. vivax parasite, PvRALP1-Ecto and PvRhopH2, were expressed and cloned to explore the persistence of naturally acquired antibody and $\mathrm{MBC}$ responses in individuals who lived in an area of low malaria endemicity in southern Thailand, using both cross-sectional and cohort study designs. The IgG antibody responses to PvRALP1-Ecto and PvRhopH2 rhoptry proteins were detected at significant levels during acute vivax malaria, predominantly of IgG1, IgG2 and IgG3 responses but these humoral responses were not maintained after infection. The prevalence of anti-PvRALP1-Ecto and -PvRhopH2 responses fell to $38.89 \%$ and $77.78 \%$ at 9 months post-infection. In contrast, the analysis of MBC responses to PvRALP1-Ecto and PvRhopH2 showed that positive rhoptry-specific MBCs persisted in a majority of individuals for at least 18 months post-infection. However, it showed no relation of these MBCs with circulating antibody responses, as around $80 \%$ of those subjects seronegative for anti-rhoptry antibodies were positive for specific MBCs. These findings suggest that whether the development and persistence of MBCs specific to rhoptry antigens will be important to antibody-based strategies of vaccine development against the blood-stage of $P$. vivax will require further study. 

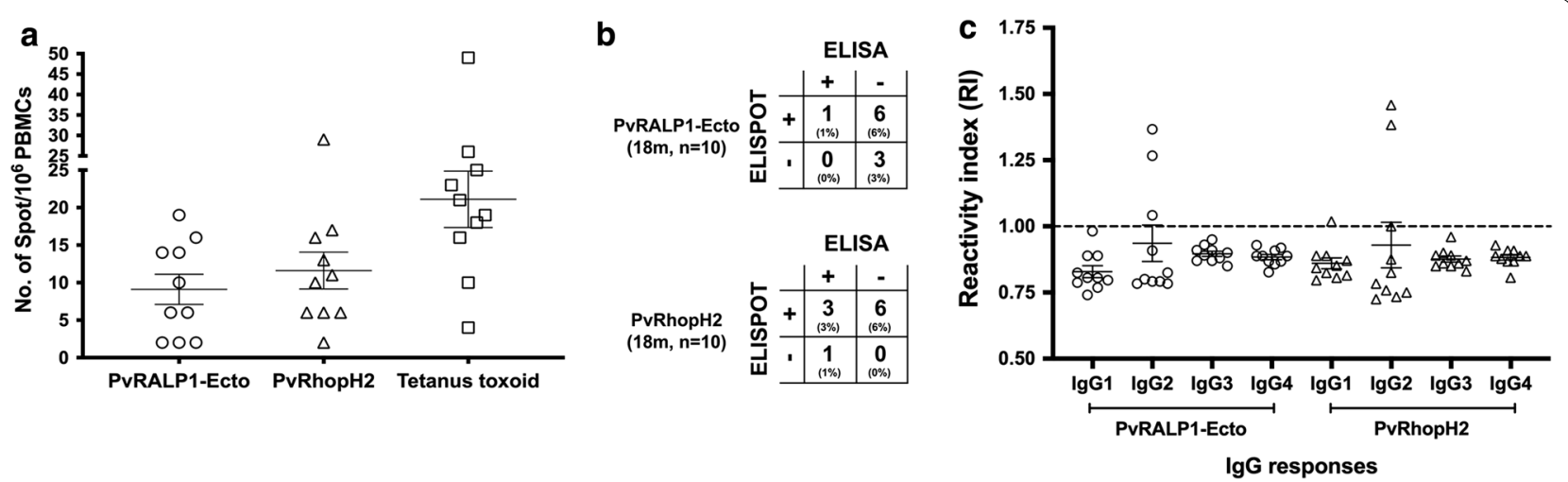

Fig. 5 Rhoptry-specific MBCs detected 18 months post-infection. a The number of specific MBC responses to the PVRALP1-Ecto and PVRhopH2 antigens, and tetanus toxoid (TT) in PBMCs from subjects 18 months after P. vivax infection were determined by ELISPOT $(n=10)$. The frequency of MBCs was expressed per million cultured PBMCs. Each symbol represents the MBC number for one individual. The line reflects the median value. $\mathbf{b}$ The correlation analysis between the responses of rhoptry-specific MBCs and antibody in 10 subjects at 18 months post-infection. $\mathbf{c}$ The comparative analysis of anti-lgG1, -lgG2, -lgG3 and -lgG4 specific to PvRALP1-Ecto and PvRhopH2 proteins in 10 subjects at 18 months post-infection. The Kruskal-Wallis one-way ANOVA test was performed to compare the mean antibody levels of the difference IgG subclasses. The horizontal line represents mean values \pm SEM. Dashed line indicates the cut-off value $(R I \geq 1)$
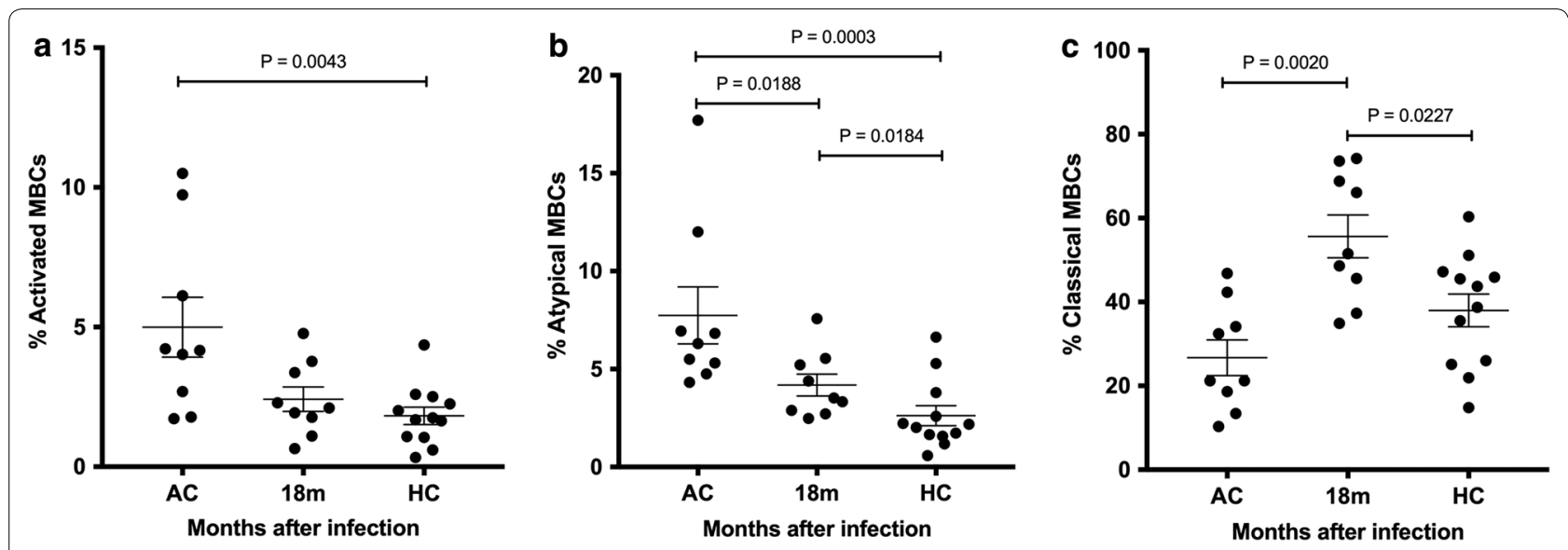

Fig. 6 The frequency of MBC subsets during acute and recovery (18 months post-infection) phases. The frequencies of (a) activated, (b) atypical and (c) classical MBCs in subjects with acute $P$. vivax infection $(A C, n=9)$ compared to post-infection $(18$ months, $n=9)$ and those of healthy controls $(\mathrm{HC}, \mathrm{n}=12)$ were determined by flow cytometric analysis. Activated $\mathrm{MBCs}\left(\mathrm{CD} 10^{-} \mathrm{CD} 19^{+} \mathrm{CD} 20^{+} \mathrm{CD} 21^{+} \mathrm{CD} 27^{+}\right)$, atypical $\mathrm{MBCs}$ $\left(\mathrm{CD} 10^{-} \mathrm{CD} 19^{+} \mathrm{CD} 20^{+} \mathrm{CD} 21^{-} \mathrm{CD} 27^{-}\right)$, classical MBCs $\left(\mathrm{CD} 10^{-} \mathrm{CD} 19^{+} \mathrm{CD} 20^{+} \mathrm{CD} 21^{-} \mathrm{CD} 27^{+}\right)$

Rhoptry proteins are among the blood-stage antigens that have been characterized and shown to induce antibody responses $[14,25]$. However, the majority of previous studies that have assessed antibody responses to rhoptry proteins used cross-sectional study designs to provide information on antibody acquisition and so propose this antibody as a serologic marker of recent exposure to $P$. vivax [44-46]. These studies lacked detailed information regarding antibody longevity and protective immunity against clinical disease, which are important in consideration of potential vaccine candidates. Here, two proteins of schizont rhoptries, PvRALP1-Ecto and PvRhopH2, were expressed to explore their ability to induce long-term antibody responses after natural $P$. vivax exposure. Approximately 66.67 and $74.36 \%$ of total patients were found to be seropositive, supporting the high seroprevalence of these antibodies in human sera from areas of Korea, Thailand and Mali [33, 35]. Furthermore, the monitored antibody responses after patient recovery from infection showed significant reduction of antibody levels 9 months post-infection. Some patients maintained these responses until 12 months in the absence of re-infection. Comparing antibody responses to these two proteins, anti-PvRhopH2 responses were 
more frequent than anti-PvRALP1-Ecto antibody responses with approximately 77.78 and $72.22 \%$ of samples seropositive 9 and 12 months post-infection, respectively. Of the 18 patients in the one-year cohort study, only one patient (Pv03) was re-infected with $P$. vivax, whereas the other patients were having their first $P$. vivax infection. Long-term antibody responses to PvRALP1Ecto and PvRhopH2 proteins could be the main immune component for protection against blood-stage vivax malaria, as previously reported with anti-RALP1, antiRhoptry associated protein 1 (RAP1), or anti-RAP2 antibodies that inhibit $P$. falciparum parasite invasion $[30,47,48]$. Moreover, the protective efficacy of antiPvRAP2 antibody was detected in the non-human Aotus primate model by showing the capacity to reduce parasitaemia after rPvRAP2 immunization [46]. However, further study of the antibodies in blocking $P$. vivax parasite invasion, as well as identification of target anti-rhoptry antibodies, will be useful for consideration of these two proteins as potential vaccine candidates.

Analysis of IgG sub-class responses to PvRALP1-Ecto and $\mathrm{PvRhopH} 2$ antigens is important for evaluating protective activity since different IgG sub-class responses mediate distinct immune effector functions [42, 43]. Also, greater understanding of the acquisition of natural immunity would strengthen the foundation of vaccine development. IgG1 and IgG3 are considered to be protective antibodies against Plasmodium spp infection through complement-mediated lysis [49] and cell-mediated mechanisms, such as opsonic phagocytosis [50-52] and antibody-dependent cellular inhibition (ADCI) [53]. In contrast, the role of IgG2 and IgG4 in protective responses has long been a matter of debate. Some studies show that IgG2 responses are mostly associated with malaria risk $[43,54,55]$ whereas a protective role of IgG2 was reported in P. falciparum exposure [56-58]. The anti-PfRESA and anti-PfMSP2 responses show potential associations with immunity despite presenting the lowest levels [58]. Here, the pattern of antibody responses to PvRALP1-Ecto and PvRhopH2 proteins were IgG1, IgG2 and IgG3 during acute malaria. Interestingly, IgG1 and IgG2 responses against these two proteins were stably maintained at post-infection, as shown by the 18 months post-infection seropositivity, whereas anti-PvRALP1-Ecto and -RhopH2-IgG3 responses did not persist. This could be because the half-life of IgG3 appears shorter than that of other IgG sub-classes [59]. Consistence with the results of other studies, blood-stage antigens of both $P$. vivax and $P$. falciparum have the ability to trigger B cells to produce cytophilic IgG1 and IgG3 [60-62] and non-cytophilic IgG2 responses [38, 60, 63]. In the present study, the development and persistence of a mixed IgG1/IgG2 response to PvRALP1-Ecto and
PvRhopH2 suggested that rhoptry proteins have some characteristic that could stimulate effector $\mathrm{T}$ cells and influence the pattern of IgG isotype switching to IgG1 and IgG2 during acute malaria and after infection. Consequently, it should benefit $P$. vivax-infected patients in killing the parasite. This conclusion comes from previous reports that cytophilic IgG1 has the high affinity to Fcy receptors [64], thus, when present, it is more effective in mediating effector mechanisms, complement-mediated lysis, opsonic phagocytosis, and antibody-dependent cellular inhibition (ADCI) $[49,50,52,53]$. In case of IgG2, it inhibits parasite invasion and blocks cyto-adherence of infected RBCs to endothelial cells, as well as activates effector cells through Fc $\gamma$ RIIa for cell-mediated immunity $[57,65]$. However, future studies are required to investigate in depth how these two IgG isotype responses to PvRALP1-Ecto and PvRhopH2 block parasite binding and invasion of red blood cells. Studies are also needed to assess the relationship between anti-rhoptry IgG1 and IgG2 antibody titres and levels of parasitaemia, degrees of prior exposure and rates of re-infection with $P$. vivax.

$\mathrm{MBCs}$ are major contributors to antibody production, but it is unclear what factors interfere with or promote maintenance of long-lived antibodies. Here, the presence of rhoptry-specific MBCs in individuals with natural $P$. vivax infections was reported. The specific MBC responses to PvRALP1-Ecto and PvRhopH2 antigens were detected at high frequency at 18 months post-infection (approximately 70 and $90 \%$ of subjects). These findings suggest the ability of PvRALP1-Ecto and $\mathrm{PvRhopH} 2$ antigens to induce MBC development during malaria infection, and their persistence in the absence of additional malaria antigen stimulation. The literature on MBCs to malaria is based on populations with various intensities of transmission. The $P$. vivax subjects who live in low malaria transmission areas of northern Thailand and northwestern Brazil appear to maintain MBC responses against malaria-specific antigens for many years after their last infection $[18,19]$. In contrast, specific MBCs were observed to be short-lived in individuals who reside in areas with high malaria transmission [20, 21]. However, the longevity of PvRALP1-Ecto and PvRhopH2-specific MBCs appears unrelated to the duration of antibody responses, as $85.71 \%$ (6 of 7) and $66.67 \%$ (6 of 9) of our vivax subjects who had long-lived MBC responses were seronegative to the respective antigen at follow up. These results suggest that circulating antibodies are likely associated with underestimates of the presence of specific MBCs in natural $P$. vivax infections. One explanation is that the alterations of circulating $\mathrm{MBC}$ sub-sets, including the expansion of atypical MBC subsets, could interfere with the development of long-lived antibody secreting cells and thus antibody production as 
this MBC sub-set in P. falciparum exposure expressed lower levels of surface IgG, possessed reduced B cell receptor signalling, and impaired antibody production in vitro $[66,67]$. However, to date, the functional significance of atypical $\mathrm{MBC}$ expansion in the acquisition and persistence of humoral immunity to malaria, as well as the factors that generate and maintain antibody-secreting cells to infections, remain unclear. Further studies to unravel a relationship between atypical MBCs and immune protection or disease progression in malaria infection are needed.

An alteration of circulating $\mathrm{MBC}$ populations was detected during malaria infection $[40,68,69]$. Here, activated and atypical MBCs were identified as the MBC sub-sets that played major roles in the responses to rhoptry proteins during acute vivax episodes. However, by 18 months post-infection these sub-sets had decreased and classical sub-sets increased. To explain these finding, it is possible that $\mathrm{CD} 27^{+}$classical MBCs were triggered via parasite infection, and class-switched into $\mathrm{CD} 27^{+}$ activated $\mathrm{MBCs}$ or $\mathrm{CD}^{2} 7^{-}$atypical MBCs, helping in parasite clearance. After infection these two MBC subclasses were reduced and class-switched back to $\mathrm{CD} 27^{+}$ classical MBCs. However, the relationship these MBC sub-sets to plasmablast/plasma cell responses, as well as that between long-lived plasma cells and antibody longevity in recovered subjects (18 months post-infection) was not demonstrated in this study. A full understanding of these relationships would be useful for the context of immunity to malaria infection and vaccine development, since generation of pre-existing antibody from longlived plasma cells could help to kill parasites. In accordance with the previous studies, the temporary changes in $\mathrm{MBC}$ sub-sets was previously reported from an area with seasonal malaria transmission $[40,68,69]$. There, malaria-specific antibodies and $\mathrm{MBCs}$ were sustained whereas the cumulative exposure to malaria gradually altered the delicate balance between circulating classical MBCs and functionally impaired atypical MBCs [69]. Despite a negative impact of atypical MBCs on the efficacy of malaria vaccines, a rare study did detect malaria-specific atypical MBCs and observed their function in natural exposure [70]. Most of MBC sub-set studies reported total populations which phenotyped as $\mathrm{CD} 19^{+} \mathrm{CD} 21^{-} \mathrm{CD} 27^{-}$(atypical MBCs) or $\mathrm{CD} 19^{+} \mathrm{CD} 27^{+}$ (classical MBCs), without any malaria specificity $[40,68$, 69]. Further studies are needed to generate a high sensitivity technique for detecting the rare populations of each malaria-specific MBC sub-set, and to study the function of atypical MBCs and their relationship to classical MBCs in circulating blood.

The limitations of this study include the lack of an independent measure of prior $P$. vivax and $P$. falciparum exposure that would be involved in the persistence of antibody and $\mathrm{MBC}$ responses. The study was also limited by the inability to recruit a larger group of $P$. vivax subjects into the cohort study. (Only 18 and 10 patients were able to be followed at 12 and 18 months in the sub-cohort 1 and 2 studies, respectively.) The observation in this study that showed persistence of long-lived PvRALP1Ecto- and PvRhopH2-specific MBCs in the absence of re-infection should be confirmed by future studies. In addition, a larger sample size would allow correlation analyses among anti-PvRALP1-Ecto and -PvRhopH2 levels, specific MBCs and frequency of MBC sub-sets.

\section{Conclusions}

The results in this study provide additional information to the available data on the characteristics of PvRALP1Ecto and PvRhopH2 proteins, and their ability to induce humoral immunity during acute malaria and after infection. The anti-PvRALP1-Ecto and -PvRhopH2 responses in the majority of individuals were short-lasting after infection. However, the specific memory B cells in a sub-group of individuals was stably detected (through 18 months). This evidence supports previous reports that effective long-lived MBCs can develop and persist in regions with low rates of malaria transmission.

\section{Supplementary information}

Supplementary information accompanies this paper at https://doi. org/10.1186/s12936-019-3009-2.

Additional file 1. PvRALP1-Ecto and PVRhopH2 specific MBC response by ELISPOT assay.

\section{Abbreviations}

MBCs: memory B cells; ASC: antibody-secreting cells; RALP1: rhoptry-associated leucine zipper-like protein 1; RhopH: high-molecular weight complex rhoptry proteins; RBC: red blood cells.

\section{Acknowledgements}

We are indebted to the malaria patients in Tha-Sae District, Chumphon Province, Thailand, for their willingness to participate in this study. This study was funded by a grant from Thailand Research Funds to PC (RSA6280064) and by a Ph.D. scholarship to PK (PHD/0162/2560). This study was supported by a National Research Foundation of Korea (NRF) grant funded by the Korea government (MSIP) (NRF-2017R1A2A2A05069562), and by the Basic Science Research Programme through the National Research Foundation of Korea (NRF) funded by the Ministry of Science, ICT and Future Planning (NRF-2015R1A4A1038666).

\section{Authors' contributions}

PK designed and planned the study; performed the experiments, statistical analysis and interpretation; and wrote the manuscript. SC wrote the manuscripts. KW performed flow cytometric analysis. SKL performed the protein expression experiments. PC and ETH designed and planned the study, and reviewed the data, analyses, interpretations, and the final draft. All authors read and approved the final manuscript.

\section{Funding}

This study was supported by a grant from Thailand Research Funds (RSA6280064) to PC and Royal Golden Jubilee Ph.D. Scholarship 
(PHD/0162/2560) to PK. This study was supported by a National Research Foundation of Korea (NRF) grant funded by the Korea government (MSIP) (NRF-2017R1A2A2A05069562) and by the Basic Science Research Programme through the National Research Foundation of Korea (NRF) funded by the Ministry of Science, ICT and Future Planning (NRF-2015R1A4A1038666).

\section{Availability of data and materials}

The datasets used and/or analysed during the current study are available from corresponding author upon reasonable request.

\section{Ethics approval and consent to participate}

Ethical approval was obtained from the Committee on Human Rights Related to Human Experimentation, Mahidol University, Thailand (MUIR 2012/079.2408). Written informed consents were taken from all of the participants. Participation in this study was entirely voluntary. All detected malaria cases were treated according to the national antimalarial treatment guidelines in Thailand. Personal information collected in this study was kept confidential.

\section{Consent for publication}

Not applicable.

\section{Competing interests}

The authors declare that they have no competing interests.

Received: 4 June 2019 Accepted: 16 November 2019

Published online: 29 November 2019

\section{References}

1. WHO. World malaria report 2018. Geneva: World Health Organization; 2018. https://www.who.int/malaria/publications/world-malaria-repor t-2018. Accessed 11 Aug 2019.

2. Howes RE, Battle KE, Mendis KN, Smith DL, Cibulskis RE, Baird JK, et al. Global epidemiology of Plasmodium vivax. Am J Trop Med Hyg. 2016;95:15-34

3. Baird JK. Malaria caused by Plasmodium vivax: recurrent, difficult to treat, disabling, and threatening to life-averting the infectious bite preempts these hazards. Pathog Glob Health. 2013;107:475-9.

4. Chu CS, White NJ. Management of relapsing Plasmodium vivax malaria. Expert Rev Anti Infect Ther. 2016;14:885-900.

5. Herrera S, Bonelo A, Perlaza BL, Fernandez OL, Victoria L, Lenis AM, et al. Safety and elicitation of humoral and cellular responses in colombian malaria-naive volunteers by a Plasmodium vivax circumsporozoite protein-derived synthetic vaccine. Am J Trop Med Hyg. 2005;73:3-9.

6. Malkin EM, Durbin AP, Diemert DJ, Sattabongkot J, Wu Y, Miura K, et al. Phase 1 vaccine trial of Pvs25H: a transmission blocking vaccine for Plasmodium vivax malaria. Vaccine. 2005;23:3131-8.

7. Payne RO, Silk SE, Elias SC, Milne KH, Rawlinson TA, Llewellyn D, et al. Human vaccination against Plasmodium vivax Duffy-binding protein induces strain-transcending antibodies. JCI Insight. 2017;2:e93683.

8. Adams JH, Sim B, Dolan SA, Fang X, Kaslow DC, Miller LH. A family of erythrocyte binding proteins of malaria parasites. Proc Natl Acad Sci USA. 1992;89:7085-9

9. Cole-Tobian JL, Michon P, Biasor M, Richards JS, Beeson JG, Mueller I, et al. Strain-specific duffy binding protein antibodies correlate with protection against infection with homologous compared to heterologous Plasmodium vivax strains in Papua New Guinean children. Infect Immun. 2009;77:4009-17.

10. Chootong P, Ntumngia FB, VanBuskirk KM, Xainli J, Cole-Tobian JL, Campbell CO, et al. Mapping epitopes of the Plasmodium vivax Duffy binding protein with naturally acquired inhibitory antibodies. Infect Immun. 2010;78:1089-95.

11. Ménard D, Barnadas C, Bouchier C, Henry-Halldin C, Gray LR, Ratsimbasoa $A$, et al. Plasmodium vivax clinical malaria is commonly observed in Duffynegative Malagasy people. Proc Natl Acad Sci USA. 2010;107:5967-71.

12. Beeson JG, Osier FHA, Engwerda CR. Recent insights into humoral and cellular immune responses against malaria. Trends Parasitol. 2008;24:578-84

13. Chang SP, Case SE, Gosnell WL, Hashimoto A, Kramer KJ, Tam LQ, Hashiro $C Q$, et al. A recombinant baculovirus 42-kilodalton C-terminal fragment of Plasmodium falciparum merozoite surface protein 1 protects Aotus monkeys against malaria. Infect Immun. 1996;64:253-61.

14. Teo A, Feng G, Brown GV, Beeson JG, Rogerson SJ. Functional antibodies and protection against blood-stage malaria. Trends Parasitol. 2016:32:887-98.

15. Wipasa J, Elliott S, Xu H, Good MF. Immunity to asexual blood stage malaria and vaccine approaches. Immunol Cell Biol. 2002;80:401-14.

16. Branch $\mathrm{OH}$, Udhayakumar $\mathrm{V}$, Hightower AW, Oloo AJ, Hawley WA, Nahlen $\mathrm{BL}$, et al. A longitudinal investigation of $\lg \mathrm{G}$ and $\lg \mathrm{M}$ antibody responses to the merozoite surface protein-1 19-kiloDalton domain of Plasmodium falciparum in pregnant women and infants: associations with febrile illness, parasitemia, and anemia. Am J Trop Med Hyg. 1998;58:211-9.

17. Arévalo-Herrera M, Forero-Peña DA, Rubiano K, Gómez-Hincapie J, Martínez NL, Lopez-Perez M, et al. Plasmodium vivax sporozoite challenge in malaria-naïve and semi-immune Colombian volunteers. PLOS ONE. 2014;9:e99754.

18. Nicolete VC, Frischmann S, Barbosa S, King CL, Ferreira MU. Naturally acquired binding-inhibitory antibodies to Plasmodium vivax Duffy binding protein and clinical immunity to malaria in rural Amazonians. J Infect Dis. 2016:214:1539-46.

19. Wipasa J, Suphavilai C, Okell LC, Cook J, Corran PH, Thaikla K, et al. Longlived antibody and B Cell memory responses to the human malaria parasites, Plasmodium falciparum and Plasmodium vivax. PLoS Pathog. 2010;6:e1000770

20. Ndungu FM, Olotu A, Mwacharo J, Nyonda M, Apfeld J, Mramba LK, et al. Memory B cells are a more reliable archive for historical antimalarial responses than plasma antibodies in no-longer exposed children. Proc Natl Acad Sci USA. 2012;109:8247-52.

21. Dorfman JR, Bejon P, Ndungu FM, Langhorne J, Kortok MM, Lowe BS, et al. B cell memory to 3 Plasmodium falciparum blood-stage antigens in a malaria-endemic area. J Infect Dis. 2005;191:1623-30.

22. Weiss GE, Traore B, Kayentao K, Ongoiba A, Doumbo S, Doumtabe D, et al. The Plasmodium falciparum-specific human memory B Cell compartment expands gradually with repeated malaria infections. PLoS Pathog. 2010;6:e1000912

23. Takala SL, Plowe CV. Genetic diversity and malaria vaccine design, testing and efficacy: preventing and overcoming 'vaccine resistant malaria'. Parasite Immunol. 2009;31:560-73.

24. Escalante AA, Lal AA, Ayala FJ. Genetic polymorphism and natural selection in the malaria parasite Plasmodium falciparum. Genetics. 1998;149:189-202.

25. Counihan NA, Kalanon M, Coppel RL, de Koning-Ward TF. Plasmodium rhoptry proteins: why order is important. Trends Parasitol. 2013;29:228-36.

26. Cowman AF, Crabb BS. Invasion of red blood cells by malaria parasites. Cell. 2006;124:755-66.

27. Alexander DL, Arastu-Kapur S, Dubremetz J-F, Boothroyd JC. Plasmodium falciparum AMA1 binds a rhoptry neck protein homologous to TgRON4, a component of the moving junction in Toxoplasma gondii. Eukaryot Cell. 2006;5:1169-73.

28. Cao J, Kaneko O, Thongkukiatkul A, Tachibana M, Otsuki H, Gao Q, et al. Rhoptry neck protein RON2 forms a complex with microneme protein AMA1 in Plasmodium falciparum merozoites. Parasitol Int. 2009;58:29-35.

29. Boothroyd JC, Dubremetz J-F. Kiss and spit: the dual roles of Toxoplasma rhoptries. Nat Rev Microbiol. 2008;6:79.

30. Ito D, Hasegawa T, Miura K, Yamasaki T, Arumugam TU, Thongkukiatkul A, Takeo S, et al. RALP1 is a rhoptry-neck erythrocyte-binding protein of Plasmodium falciparum merozoite and a potential blood-stage vaccine candidate antigen. Infect Immun. 2013;81:4290-8.

31. Kaneko O. Erythrocyte invasion: vocabulary and grammar of the Plasmodium rhoptry. Parasitol Int. 2007;56:255-62.

32. Ling IT, Kaneko O, Narum DL, Tsuboi T, Howell S, Taylor HM, et al. Characterisation of the rhoph2 gene of Plasmodium falciparum and Plasmodium yoelii. Mol Biochem Parasitol. 2003;127:47-57.

33. Wang B, Lu F, Cheng Y, Li J, Ito D, Sattabongkot J, et al. Identification and characterization of the Plasmodium falciparum $\mathrm{RhopH} 2$ ortholog in Plasmodium vivax. Parasitol Res. 2013;112:585-93.

34. Haase S, Cabrera A, Langer C, Treeck M, Struck N, Herrmann S, et al. Characterization of a conserved rhoptry-associated leucine zipper-like protein in the malaria parasite Plasmodium falciparum. Infect Immun. 2008;76:879-87. 
35. Cheng Y, Li J, Ito D, Kong D-H, Ha K-S, Lu F, et al. Antigenicity and immunogenicity of PVRALP1, a novel Plasmodium vivax rhoptry neck protein. Malar J. 2015;14:186.

36. López C, Yepes-Pérez Y, Diaz-Arevalo D, Patarroyo ME, Patarroyo MA. The in vitro antigenicity of Plasmodium vivax rhoptry neck protein 2 (PvRON2) B-and T-epitopes selected by HLA-DRB1 binding profile. Front Cell Infect Microbiol. 2018;8:156.

37. Moreno-Perez DA, Montenegro M, Patarroyo ME, Patarroyo MA. Identification, characterization and antigenicity of the Plasmodium vivax rhoptry neck protein 1 (Pv RON1). Malar J. 2011;10:314.

38. Changrob S, Wang B, Han J-H, Lee S-K, Nyunt MH, Lim CS, et al. Naturallyacquired immune response against Plasmodium vivax rhoptry-associated membrane antigen. PLoS ONE. 2016;11:e0148723.

39. Tsuboi T, Takeo S, Iriko H, Jin L, Tsuchimochi M, Matsuda S, et al. Wheat germ cell-free system-based production of malaria proteins for discovery of novel vaccine candidates. Infect Immun. 2008;76:1702-8.

40. Changrob S, McHenry AM, Nyunt MH, Sattabongkot J, Han E-T, Adams JH, et al. Persistence of long-lived memory B cells specific to Duffy binding protein in individuals exposed to Plasmodium vivax. Sci Rep. 2018;8:8347.

41. Min HMK, Changrob S, Soe PT, Han JH, Muh F, Lee S-K, et al. Immunogenicity of the Plasmodium vivax merozoite surface protein 1 paralog in the induction of naturally acquired antibody and memory B cell responses. Malar J. 2017;16:354.

42. Bouharoun-Tayoun H, Attanath P, Sabchareon A, Chongsuphajaisiddhi T, Druilhe P. Antibodies that protect humans against Plasmodium falciparum blood stages do not on their own inhibit parasite growth and invasion in vitro, but act in cooperation with monocytes. J Exp Med. 1990;172:1633-41.

43. Bouharoun-Tayoun H, Druilhe P. Plasmodium falciparum malaria: evidence for an isotype imbalance which may be responsible for delayed acquisition of protective immunity. Infect Immun. 1992;60:1473-81.

44. Patarroyo MA, Perez-Leal O, Lopez Y, Cortes J, Rojas-Caraballo J, Gomez A, et al. Identification and characterisation of the Plasmodium vivax rhoptryassociated protein 2. Biochem Biophys Res Commun. 2005;337:853-9.

45. Perez-Leal O, Mongui A, Cortes J, Yepes G, Leiton J, Patarroyo MA. The Plasmodium vivax rhoptry-associated protein 1. Biochem Biophys Res Commun. 2006;341:1053-8

46. Rojas-Caraballo J, Mongui A, Giraldo MA, Delgado G, Granados D, Millan-Cortes $D$, et al. Immunogenicity and protection-inducing ability of recombinant Plasmodium vivax rhoptry-associated protein 2 in Aotus monkeys: a potential vaccine candidate. Vaccine. 2009;27:2870-6.

47. Howard RF, Jacobson KC, Rickel E, Thurman J. Analysis of inhibitory epitopes in the Plasmodium falciparum rhoptry protein RAP-1 including identification of a second inhibitory epitope. Infect Immun. 1998;66:380-6.

48. Stowers AW, Cooper JA, Ehrhardt T, Saul A. A peptide derived from a B cell epitope of Plasmodium falciparum rhoptry associated protein 2 specifically raises antibodies to rhoptry associated protein 1. Mol Biochem Parasitol. 1996:82:167-80.

49. Boyle MJ, Reiling L, Feng G, Langer C, Osier FH, Aspeling-Jones H, et al. Human antibodies fix complement to inhibit Plasmodium falciparum invasion of erythrocytes and are associated with protection against malaria. Immunity. 2015:42:580-90.

50. Hill DL, Eriksson EM, Li Wai Suen CSN, Chiu CY, Ryg-Cornejo V, Robinson $L$, et al. Opsonising antibodies to P. falciparum merozoites associated with immunity to clinical malaria. PLoS One. 2013;8:e74627.

51. Osier FH, Feng G, Boyle MJ, Langer C, Zhou J, Richards JS, et al. Opsonic phagocytosis of Plasmodium falciparum merozoites: mechanism in human immunity and a correlate of protection against malaria. BMC Med. 2014;12:108

52. Hill DL, Wilson DW, Sampaio NG, Eriksson EM, Ryg-Cornejo V, Harrison GLA, et al. Merozoite antigens of Plasmodium falciparum elicit straintranscending opsonizing immunity. Infect Immun. 2016;84:2175-84

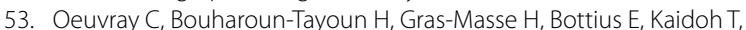
Aikawa M, et al. Merozoite surface protein-3: a malaria protein inducing antibodies that promote Plasmodium falciparum killing by cooperation with blood monocytes. Blood. 1994;84:1594.
54. Schreiber N, Brattig N, Evans J, Tsiri A, Horstmann RD, May J, et al. Cerebral malaria is associated with $\lg G 2$ and $\lg G 4$ antibody responses to recombinant Plasmodium falciparum RIFIN antigen. Microb Infect. 2006:8:1269-76.

55. Groux H, Gysin J. Opsonization as an effector mechanism in human protection against asexual blood stages of Plasmodium falciparum: functional role of IgG subclasses. Res Immunol. 1990;141:529-42.

56. Reiling L, Richards JS, Fowkes FJl, Barry AE, Triglia T, Chokejindachai W, et al. Evidence that the erythrocyte invasion ligand PfRh2 is a target of protective immunity against Plasmodium falciparum malaria. J Immunol. 2010;185:6157-67.

57. Deloron P, Dubois B, Le Hesran JY, Riche D, Fievet N, Cornet M, et al. Isotypic analysis of maternally transmitted Plasmodium falciparum-specific antibodies in Cameroon, and relationship with risk of $P$ falciparum infection. Clin Exp Immunol. 1997;110:212-8.

58. Aucan C, Traoré Y, Tall F, Nacro B, Traoré-Leroux T, Fumoux F, et al. High immunoglobulin G2 (lgG2) and low lgG4 levels are associated with human resistance to Plasmodium falciparum malaria. Infect Immun. 2000;68:1252.

59. Morell A, Terry WD, Waldmann TA. Metabolic properties of lgG subclasses in man. J Clin Invest. 1970;49:673-80.

60. França CT, He W-Q, Gruszczyk J, Lim NTY, Lin E, Kiniboro B, et al. Plasmodium vivax reticulocyte binding proteins are key targets of naturally acquired immunity in young Papua New Guinean Children. PLoS Negl Trop Dis. 2016;10:e0005014.

61. Rouhani M, Zakeri S, Mehrizi AA, Djadid ND. Comparative analysis of the profiles of lgG subclass-specific responses to Plasmodium falciparum apical membrane antigen-1 and merozoite surface protein-1 in naturally exposed individuals living in malaria hypoendemic settings, Iran. Malar J. 2015; 14:58.

62. Zakeri S, Babaeekhou L, Mehrizi AA, Abbasi M, Djadid ND. Antibody responses and avidity of naturally acquired anti-Plasmodium vivax Duffy binding protein (PVDBP) antibodies in individuals from an area with unstable malaria transmission. Am J Trop Med Hyg. 2011;84:944-50.

63. Kwenti TE, Kukwah TA, Kwenti TDB, Nyassa BR, Dilonga MH, Enow-Orock $\mathrm{G}$, et al. Comparative analysis of lgG and lgG subclasses against Plasmodium falciparum MSP-119 in children from five contrasting bioecological zones of Cameroon. Malar J. 2019;18:16.

64. Vidarsson G, Dekkers G, Rispens T. IgG subclasses and allotypes: from structure to effector functions. Front Immunol. 2014;5:520.

65. Warmerdam PA, van de Winkel JG, Vlug A, Westerdaal NA, Capel PJ. A single amino acid in the second lg-like domain of the human Fc gamma receptor II is critical for human IgG2 binding. J Immunol. 1991;147:1338.

66. Portugal S, Tipton CM, Sohn H, Kone Y, Wang J, Li S, et al. Malaria-associated atypical memory $B$ cells exhibit markedly reduced $B$ cell receptor signaling and effector function. eLife. 2015;4:e07218.

67. Sullivan RT, Kim CC, Fontana MF, Feeney ME, Jagannathan P, Boyle MJ, et al. FCRL5 delineates functionally impaired memory b cells associated with Plasmodium falciparum Exposure. PLoS Pathog. 2015;11:e1004894.

68. Ayieko C, Maue AC, Jura WGZO, Noland GS, Ayodo G, Rochford R, et al. Changes in B cell populations and merozoite surface protein-1-specific memory $B$ cell responses after prolonged absence of detectable P. falciparum infection. PLoS One. 2013:8:e67230.

69. Soares RR, Cunha CF, Ferraz-Nogueira R, Marins-dos-Santos A, Rodriguesda-Silva RN, da Silva Soares I, et al. Apical membrane protein 1-specific antibody profile and temporal changes in peripheral blood B-cell populations in Plasmodium vivax malaria. Parasite Immunol. 2019;41:e12662.

70. Krishnamurty AT, Thouvenel CD, Portugal S, Keitany GJ, Kim KS, Holder A, et al. Somatically hypermutated Plasmodium-specific $\lg M(+)$ memory B cells are rapid, plastic, early responders upon malaria rechallenge. Immunity. 2016;45:402-14.

\section{Publisher's Note}

Springer Nature remains neutral with regard to jurisdictional claims in published maps and institutional affiliations. 